\title{
Pain Physician Protocols and Promise for a Unified Approach for the Delivery of Interventional Pain Management
}

Curtis W.Slipman, MD

Two thousand three begins with a continuation of many of the difficult and frustrating issues that have been thrust upon interventional pain physicians for the last several years. These are all well known to each of you; a steady and insidious decline in reimbursement, attempts to eliminate payment for worthwhile procedures, delay tactics precluding new interventions from becoming a component of our pain modulation armamentarium, an exponential increase in rules and regulations governing the delivery of healthcare, doubling and tripling of malpractice premiums, and litigation that is predicated on our ability to provide perfect outcomes rather than accepted and carefully considered interventions that have risks. Certainly this listing is not exhaustive, except in the sense that they could exhaust any reasonable person. So how are we confronting these challenges? I am proud to say we are doing it as professionals. That is, we have not lost our focus of delivering the best quality care possible as evidenced by the quality and spectrum of articles contained in this quarterly issue.

Windsor shares the results of original work in which he attempts to refine the technique of medial branch radiofrequency ablation. He provides unique insights that can be used by others to further develop his ideas. Chandler similarly provides new information in his retrospective

From the Editor-in-chief of Pain Physician. Dr. Slipman is Director at Penn Spine Center and associate professor Department of Rehabilitation Medicine at University of Pennsylvania Medical Center. Address correspondence: Curtis W. Slipman, MD, The Penn Spine Center, Ground Floor White Bldg., 3400 Spruce Street, Philadelphia, PA 19104. E-mail: slipman@mail.med.upenn.edu assessment of patients receiving spinal cord stimulation for spinal stenosis. Lenrow and Chou offer us their perspective concerning randomized control studies. In their article an eloquent description of the hierarchal placement of various types of research protocols in spine interventions is developed. They go on to critically examine the advantages and limitations of randomized control trials (RCT), while consistently weaving in an ethical theme. In particular, they explore practical matters related to providing sham interventions or placebo controls. Much of this manuscript is thought provoking as definitive answers are not available, however they highlight one aspect that is universally accepted, the strength of specific study designs. Lenrow and Chou remind us that systematic reviews are the most clinically relevant and can have greater weight than single studies provided they are done properly.

It is with such careful consideration that the updated evidence-based practice guidelines for interventional techniques in the management of chronic spinal pain manuscript has been coordinated by Manchikanti et al. Prior to revising these interventional clinical guidelines a methodical analyses of the historical aspects of the development of guidelines, a critical appraisal of prior government and independent sponsored evidenced based medicine studies, and the implementation of a systematic mechanism by which this should be affected was conducted by Manchikanti, Heavner, Racz, Mekhail, Schultz, Hansen, and Singh. The scientific basis for and details of this methodical review process is delineated in this issue. Manchikanti et al, provided us with a straightforward and generally accepted mechanism to analyze the literature pertaining to interventional pain management. Using this superb and valuable evaluative process provides the structure from which clinical guidelines can be developed, which was accomplished. Indeed, the real gem in this issue is the monumental manuscript containing updated clinical guidelines for interventional pain management.

The 2003 revised guidelines offers each reader a wealth of information regarding an expansive list of diagnosis, tests, and treatments. As can be readily discerned by reviewing the list of contributors, the reader has been given the consensus opinion of numerous experienced, knowledgeable interventional physicians who incorporated the aforementioned systematic analyses. Even though the perspective of each individual physician is quite unique, agreement among the authors was secured, which in and of itself is a huge task. If these guidelines are perused with the expectation that they be incorporated into daily practice, then the reader must remember that in the final analysis there is no singular answer to every pain problem. These are guidelines and not absolute standards or rules.

I thank all of the contributors to this issue and, in particular, the developers of the revised guidelines for demonstrating extraordinary leadership. Interventional pain physicians are doing their utmost to incorporate scientific methodology for the diagnosis and treatment of a myriad of painful conditions. These efforts persist in the face of a growing list of deliberate daunting external influences. We should be appreciative and proud. 
